\title{
Photochemical \&

\section{Incorporation of a high potential quinone reveals that electron transfer in Photosystem I becomes highly asymmetric at low temperature $\dagger$}

\author{
Sam Mula, ${ }^{a}$ Anton Savitsky, ${ }^{* b}$ Klaus Möbius, ${ }^{b, c}$ Wolfgang Lubitz, ${ }^{b}$ John H. Golbeck, ${ }^{d, e}$ Mahir D. Mamedov, ${ }^{f}$ \\ Alexey Yu. Semenov* ${ }^{*}$ and Art van der Est*a
}

Received 7th October 2011, Accepted 6th December 2011

DOI: $10.1039 / \mathrm{c} 2 \mathrm{pp} 05340 \mathrm{c}$

Photosystem I (PS I) has two nearly identical branches of electron-transfer co-factors. Based on point mutation studies, there is general agreement that both branches are active at ambient temperature but that the majority of electron-transfer events occur in the A-branch. At low temperature, reversible electron transfer between $\mathrm{P}_{700}$ and $\mathrm{A}_{1 \mathrm{~A}}$ occurs in the A-branch. However, it has been postulated that irreversible electron transfer from $\mathrm{P}_{700}$ through $\mathrm{A}_{1 \mathrm{~B}}$ to the terminal iron-sulfur clusters $\mathrm{F}_{\mathrm{A}}$ and $\mathrm{F}_{\mathrm{B}}$ occurs via the $\mathrm{B}$ branch. Thus, to study the directionality of electron transfer at low temperature, electron transfer to the iron-sulfur clusters must be blocked. Because the geometries of the donor-acceptor radical pairs formed by electron transfer in the A- and B-branch differ, they have different spin-polarized EPR spectra and echo-modulation decay curves. Hence, time-resolved, multiple-frequency EPR spectroscopy, both in the direct-detection and pulse mode, can be used to probe the use of the two branches if electron transfer to the iron-sulfur clusters is blocked. Here, we use the PS I variant from the menB deletion mutant strain of Synechocyctis sp. PCC 6803, which is unable to synthesize phylloquinone, to incorporate 2,3-dichloro1,4-naphthoquinone $\left(\mathrm{Cl}_{2} \mathrm{NQ}\right)$ into the $\mathrm{A}_{1 \mathrm{~A}}$ and $\mathrm{A}_{1 \mathrm{~B}}$ binding sites. The reduction midpoint potential of $\mathrm{Cl}_{2} \mathrm{NQ}$ is approximately $400 \mathrm{mV}$ more positive than that of phylloquinone and is unable to transfer electrons to the iron-sulfur clusters. In contrast to previous studies, in which the iron-sulfur clusters were chemically reduced and/or point mutations were used to prevent electron transfer past the quinones, we find no evidence for radical-pair formation in the B-branch. The implications of this result for the directionality of electron transfer in PS I are discussed.

\section{Introduction}

In Photosystem I (PS I) the PsaA and PsaB protein subunits bind two branches of electron-transfer cofactors extending from $\mathrm{P}_{700}$, a chlorophyll $a$ /chlorophyll $a^{\prime}$ dimer, on the lumenal side of the thylakoid membrane to the iron-sulfur cluster $\mathrm{F}_{\mathrm{X}}$ on the stromal side (Fig. 1). Each of the two branches carries two monomeric chlorophyll $a$ molecules, $\mathrm{A}_{-1 \mathrm{~A}(\mathrm{~B})}$ and $\mathrm{A}_{0 \mathrm{~A}(\mathrm{~B})}$, and a phylloquinone molecule, $\mathrm{A}_{1 \mathrm{~A}(\mathrm{~B})}$, where $\mathrm{A}$ and $\mathrm{B}$ indicate the protein

\footnotetext{
${ }^{a}$ Department of Chemistry, Brock University, St. Catharines, ON, Canada.E-mail: avde@brocku.ca; Fax: +1 9056829020; Tel: +1 9056885550

${ }^{b}$ Max-Planck-Institut für Bioanorganische Chemie, 45470 Mülheim an der Ruhr, Germany. E-mail: savitsky@mpi-muelheim.mpg.de; Fax: +49 2083063955; Tel: +492083063555

${ }^{c}$ Department of Physics, Free University Berlin, 14195 Berlin, Germany ${ }^{d}$ Department of Biochemistry and Molecular Biology, The Pennsylvania State University, University Park, Pennsylvania, 16802, USA

${ }^{e}$ Department of Chemistry, The Pennsylvania State University, University Park, Pennsylvania, 16802, USA

${ }^{A}$ A.N. Belozersky Institute of Physical-Chemical Biology, Moscow State

University, Moscow, 119992 Leninskie Gory, Russia.E-mail: semenov@ genebee.msu.ru; Fax: + 7 4959393181; Tel: + 74959393188

$\dagger$ This article is published as part of a themed issue in honour of Professor Kurt Schaffner on the occasion of his 80th birthday.
}

subunit PsaA or PsaB to which the cofactor is mainly bound. The terminal iron-sulfur clusters, $\mathrm{F}_{\mathrm{A}}$ and $\mathrm{F}_{\mathrm{B}}$, are both bound to the protein subunit PsaC. Upon light excitation at room temperature, electrons are transferred from $\mathrm{P}_{700}$ to $\mathrm{F}_{\mathrm{B}}$ via the intervening cofactors. Although there is some debate about whether the initial charge separation occurs independently in each branch between $\mathrm{A}_{-1}$ and $\mathrm{A}_{0}$, ${ }^{1}$ or between $\mathrm{P}_{700}$ and $\mathrm{A}_{0}$ considered as a loosely bound dimer of $\mathrm{A}_{-1 \mathrm{~A}(\mathrm{~B})}$ and $\mathrm{A}_{0 \mathrm{~A}(\mathrm{~B})}$ chlorophyll molecules, ${ }^{2}$ there is general consensus that both branches of cofactors are active in electron transfer. This conclusion has been reached primarily from point-mutation studies, which show that at room temperature the $200 \mathrm{~ns}$ phase of $\mathrm{A}_{1}$ to $\mathrm{F}_{\mathrm{X}}$ electron transfer occurs in the A-branch while the 20 ns phase occurs in the B-branch. ${ }^{3-9}$

In contrast to the behavior in PS I, electron transfer in reaction centers from purple bacteria and Photosystem II is unidirectional along the A-branch and extremely difficult to re-direct into the inactive B-branch. This difference appears to be related to the fact that in type II reaction centers electron transfer proceeds from the A-branch quinone to the B-branch quinone, which exchanges with the quinone pool following double reduction and protonation. In Photosystem I, on the other hand, both branches of cofactors converge at $\mathrm{F}_{\mathrm{X}}$ and there is no obvious functional advantage to uni-directionality or bi-directionality. At present the 


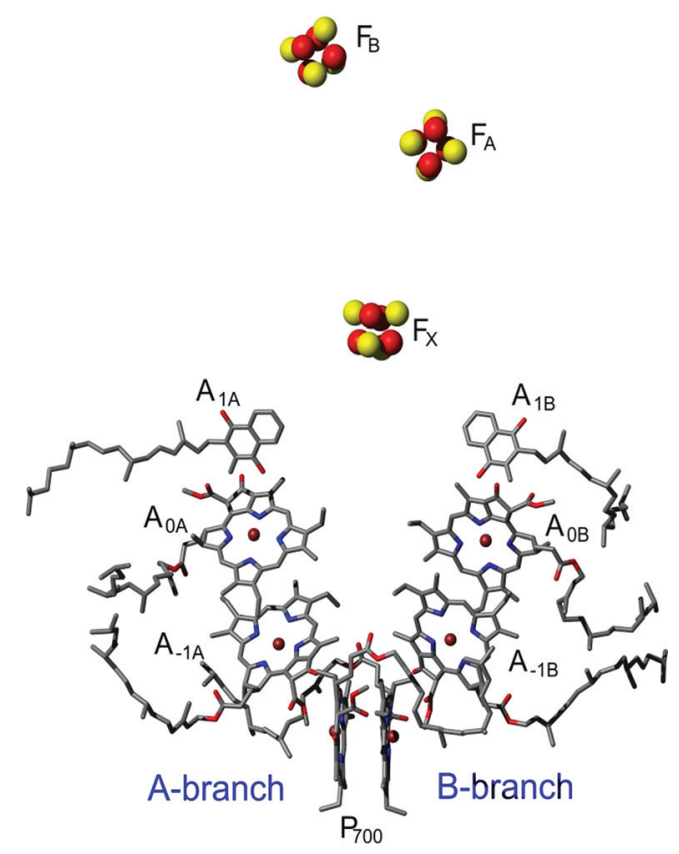

Fig. 1 Structural arrangement of the electron-transfer cofactors in Photosystem I. The positions of the cofactors are from the $2.5 \AA$ resolution X-ray structure ${ }^{60}$ (PDB entry $\left.1 \mathrm{JB} 0\right)$.

factors that determine the relative use of the two branches and lead to such a large difference between type I and type II reaction centers are not well understood. In this context it is of interest to study the temperature dependence of the directionality. In type II reaction centers, the very strong bias towards the Abranch of the electron transfer does not depend on temperature. In PS I, the relative electron-transfer activity of the two branches below the glass-transition temperature of the protein at about $200 \mathrm{~K}$ is less certain because the kinetic behavior becomes heterogeneous. At least two fractions of PS I complexes are observed in which either irreversible electron transfer to $\mathrm{F}_{\mathrm{A}}$ and $F_{B}$, or reversible electron transfer to $A_{1}$ and/or $F_{X}$ occurs. ${ }^{10}$ Electron transfer in the A-branch from $A_{1 A}$ to $F_{X}$ is strongly activated, ${ }^{11}$ while the corresponding step in the $\mathrm{B}$-branch is nearly activationless. ${ }^{12}$ Consistent with this difference in the activation energies, low-temperature EPR studies of branch-specific point mutants show that reversible electron transfer to $A_{1}$ occurs exclusively in the A-branch. ${ }^{6,8,13-15}$ However, this observation does not answer the question of the directionality at low temperature because the pathway leading to irreversible charge separation remains unclear.

To address this issue it is necessary to prevent electron transfer past $A_{1}$ so that irreversible charge separation does not occur. A number of studies have been carried out in which forward electron transfer past $A_{1}$ has been blocked by prereduction of the iron-sulfur clusters ${ }^{4,13,16-21}$ and/or in which A-branch electron transfer is partially hindered by mutation of $\mathrm{M} 688_{\mathrm{PsaA}}$, which provides the axial ligand to $\mathrm{A}_{0 \mathrm{~A}} \cdot{ }^{21,22}$ Under these conditions, additional components with different kinetic and spectral properties are observed in the time-resolved EPR signals from $\mathrm{P}_{700}^{+} \mathrm{A}_{1}^{\cdot-}$. Modeling of the spin-polarized EPR spectra and outof-phase electron-spin echo-envelope modulation (ESEEM) curves suggest that under reducing conditions, or when electron transfer in the A-branch is hindered, components arising from both the $\mathrm{A}-$ and $\mathrm{B}-$ branch radical pairs $\mathrm{P}_{700}^{+} \mathrm{A}^{\cdot-} \mathrm{A}$ and $\mathrm{P}_{700}^{+{ }_{70}} \mathrm{~A}^{\cdot-}$ are observed. These results have been interpreted as indicating that the electron transfer is also bidirectional at low temperature. ${ }^{21}$ However, it has also been suggested that the electron transfer is highly biased towards the A-branch. ${ }^{14,22}$ Another method of preventing electron transfer past $A_{1}$ is to inhibit the assembly of $\mathrm{F}_{\mathrm{X}}$. It has been shown that deletion of the $\mathrm{rubA}$ gene of Synechocystis sp. PCC 6803, which encodes for a rubredoxin that is involved in the assembly of PS I, results in complexes lacking the iron-sulfur clusters $F_{A}, F_{B}$ and $F_{X}$ in which no irreversible electron transfer is observed at low temperature. ${ }^{23,24}$ In contrast to the prereduced samples, the low-temperature timeresolved EPR spectra and echo-modulation curves of PS I complexes from the $r u b A$ variant are indistinguishable from the wild type and show no evidence for electron transfer in the Bbranch. $^{6,23}$

The difference in behavior between the prereduced samples and the $r u b A$ variant suggests that the directionality may be altered by either reduction or removal of $F_{X}$. Indeed it has been shown that electrons can be re-directed into the B-branch by mutations $^{25}$ and/or harsh solubilization methods. ${ }^{26}$ Thus, it is conceivable that either reduction at high $\mathrm{pH}$ or the absence of the iron-sulfur clusters could alter the directionality. Here we address this issue by incorporating the high potential quinone, 2,3-dichloro-1,4-naphthoquinone $\left(\mathrm{Cl}_{2} \mathrm{NQ}\right)$ into the $\mathrm{A}_{1}$ binding sites of PS I to prevent forward electron transfer to the ironsulfur clusters. To incorporate $\mathrm{Cl}_{2} \mathrm{NQ}$ we use PS I from the $m e n B$ variant of Synechocystis sp. PCC 6803. Inactivation of the $m e n B$ gene, which codes for a naphthoate synthase, inhibits the biosynthesis of phylloquinone ${ }^{27}$ and in its absence, plastoquinone- 9 binds to the $\mathrm{A}_{1 \mathrm{~A}}$ and $\mathrm{A}_{1 \mathrm{~B}}$ sites. ${ }^{28}$ Because plastoquinone9 binds relatively weakly, it can be displaced by incubation with a wide variety of naphthoquinones. ${ }^{29-33}$ The structures of phylloquinone, plastoquinone- 9 and $\mathrm{Cl}_{2} \mathrm{NQ}$ are shown in Fig. 2 along with their first reduction midpoint potentials in dimethylformamide (DMF). The reduction potential of $\mathrm{Cl}_{2} \mathrm{NQ}$ in DMF is more than $400 \mathrm{mV}$ more positive than that of phylloquinone, and the premise of the experiments presented here is that a similar difference in the potentials of phylloquinone and $\mathrm{Cl}_{2} \mathrm{NQ}$ would be

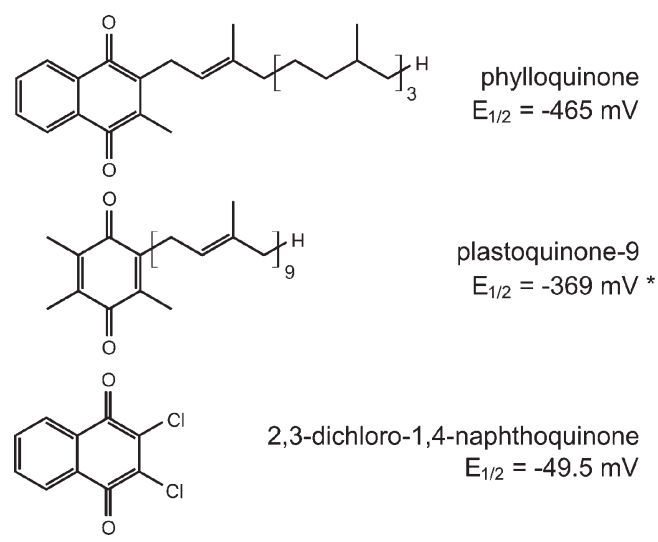

Fig. 2 Structures of phylloquinone, plastoquinone- 9 and $\mathrm{Cl}_{2} \mathrm{NQ}$ and their first reduction midpoint potentials in DMF versus the normal hydrogen electrode. $(*)$ The midpoint potential for plastoquinone-9 is taken from the literature. ${ }^{61}$ 
expected in the $\mathrm{A}_{1 \mathrm{~A}}$ and $\mathrm{A}_{1 \mathrm{~B}}$ binding sites. Estimates of the midpoint potential of the B-branch phylloquinone place it $25 \mathrm{mV}^{34}$ $155 \mathrm{mV}^{35}$ or $173 \mathrm{mV}^{36}$ more negative than the A-branch phylloquinone. If our premise is correct, electron transfer from $\mathrm{Cl}_{2} \mathrm{NQ}$ to $F_{X}$ in both branches should require a larger activation energy than the phylloquinone to $F_{X}$ transfer in the A-branch of native PS I.

The main advantage of blocking electron transfer in this way is that neither prereduction nor removal of the iron-sulfur clusters are required and, hence, possible changes in the structure, electrostatic environment, protonation state of the protein, etc. should be minimized. We will show that the multi-frequency timeresolved EPR data from these samples are consistent with unidirectional electron transfer in the A-branch and do not show any of the characteristic features that have been associated with the $\mathrm{B}-$ branch radical pair $\mathrm{P}_{700}^{\cdot+} \mathrm{A}_{1 \mathrm{~B}}^{\cdot-}$.

\section{Materials and methods}

\section{Growth and isolation of PS I from the menB and menB/rubA mutants}

The menB deletion mutant strain of Synechocystis sp. PCC 6803 was grown under low light, and trimeric PS I complexes were isolated as described previously. ${ }^{27}$ The isolated trimers were brought to a chlorophyll $a$ concentration of $2 \mathrm{mg} \mathrm{mL}^{-1}$ by centrifuging to a thick paste using $100 \mathrm{~K}$ Ultracell purification membranes followed by resuspension in a $50 \mathrm{mM}$ Tris-buffer at $\mathrm{pH}$ 8.0 containing $0.05 \%$ n-dodecyl- $\beta$-D-maltoside and $1 \%$ glycerol as a cryo-protectant. The menB/rubA mutant strain of Synechococcus sp. PCC 7002 was grown and thylakoid membrane fragments were prepared as described previously. ${ }^{37}$ The thylakoid suspension was centrifuged to a chlorophyll $a$ concentration of $16 \mathrm{mg} \mathrm{mL}^{-1}$. Photosystem I particles were not isolated from the thylakoids to avoid detergent-induced structural changes.

\section{Incubation of PS I with $\mathrm{Cl}_{2} \mathrm{NQ}$}

1,2-Dichloro-1,4-naphthoquinone was obtained from SigmaAldrich Chemicals and was dissolved in DMSO. For the menB trimers, a $10 \mu \mathrm{L}$ aliquot of a $20 \mathrm{mM} \mathrm{Cl}_{2} \mathrm{NQ}$ solution was added to approximately $100 \mu \mathrm{L}$ of a PS I trimer solution at a chlorophyll $a$ concentration of $2 \mathrm{mg} \mathrm{mL}{ }^{-1}$. The samples were incubated for one hour on ice before being washed with one $4 \mathrm{~mL}$ aliquot of buffer and centrifuged to a thick paste followed by resuspension to a volume of $150 \mu \mathrm{L}$.

The menB/rubA thylakoid suspension was incubated with $5 \mu \mathrm{L}$ of a $4 \mathrm{mM}$ solution of $\mathrm{Cl}_{2} \mathrm{NQ}$ for $1 \mathrm{~h}$. This process was carried out in low light to reduce damage to the double mutant.

\section{Cyclic voltammetry of quinones in DMF}

Reduction-wave voltammograms of phylloquinone and $\mathrm{Cl}_{2} \mathrm{NQ}$ were measured using a BAS Epsilon and C3 Cell Stand (Bioanalytical Systems) with a platinum working electrode, a $3 \mathrm{M} \mathrm{NaCl}$ $\mathrm{Ag} / \mathrm{AgCl}$ reference electrode and a platinum auxiliary electrode. The samples were prepared by dissolving $\sim 5 \mathrm{mg}$ of the quinones and $0.15 \mathrm{~g}$ of the supporting electrolyte tetrabutylammonium- hexafluorate-phosphate in $10 \mathrm{~mL}$ of dry DMF. The solutions were bubbled with nitrogen gas for five minutes to remove oxygen. Voltammograms were cycled between $+250 \mathrm{mV}$ and $-2000 \mathrm{mV}$.

\section{Time-resolved optical spectroscopy in the near-infrared region}

The decay of $\mathrm{P}_{700}^{++}$due to charge recombination was measured at $820 \mathrm{~nm}$ using a laboratory-built time-resolved spectrophotometer. The samples were diluted to a chlorophyll concentration of $50 \mu \mathrm{g} \mathrm{mL}^{-1}$ and placed in a quartz cuvette with a path length of $10 \mathrm{~mm}$. The samples contained $10 \mathrm{mM}$ sodium ascorbate, $4 \mu \mathrm{M}$ DCPIP as an exogenous donor and were prepared in an anaerobic chamber with an atmosphere of $10 \%$ hydrogen and $90 \%$ nitrogen. The kinetic traces were analyzed by fitting a multiexponential decay using the Marquardt least-squares algorithm programmed in the Igor Pro language (Wavemetrics).

\section{Time-resolved optical spectroscopy at $700 \mathrm{~nm}$}

The samples were diluted to a chlorophyll concentration of $10 \mu \mathrm{g} \mathrm{mL}^{-1}$. The samples contained $5 \mathrm{mM}$ sodium ascorbate, $4 \mu \mathrm{M}$ DCPIP as an exogenous donor and were prepared under aerobic conditions.

\section{Time-resolved and CW EPR experiments at X-band and Q-band}

Samples for the EPR experiments at X- and Q-band contained $1 \mathrm{mM}$ sodium ascorbate and $50 \mu \mathrm{M}$ phenazine methosulfate as an external electron donor and were dark adapted for $20 \mathrm{~min}$ before being frozen in the dark. The EPR experiments were carried out using a Bruker Elexsys E580 spectrometer or a modified Bruker ER 200D-SRC spectrometer as described previously. ${ }^{38}$ For the time-resolved EPR (TREPR, direct detection without field modulation or echo-detected pulse EPR) experiments the samples were illuminated at $532 \mathrm{~nm}$ and $10 \mathrm{~Hz}$ using a Nd:YAG laser (Continuum Surelite). To determine the throughput of electrons to the terminal iron-sulfur clusters at low temperature the samples were frozen to $15 \mathrm{~K}$ in the dark and then illuminated continuously for five minutes with white light from a Schott KL-1500 visible wavelength lamp with an intensity of $1.3 \mu \mathrm{E} \mathrm{s}^{-1}$ to accumulate $\left(\mathrm{F}_{\mathrm{A}} / \mathrm{F}_{\mathrm{B}}\right)^{-}$. The CW-EPR spectra of $\left(\mathrm{F}_{\mathrm{A}} / \mathrm{F}_{\mathrm{B}}\right)^{-}$were collected using $100 \mathrm{kHz}$ field modulation with an amplitude of $1.0 \mathrm{mT}$ and a nominal microwave power of $1.0 \mathrm{~mW}$. The spectrum taken before illumination was subtracted to remove background signals from the resonator.

X-band out-of-phase ESEEM curves were collected at $80 \mathrm{~K}$ on the Bruker Elexsys E580 spectrometer. The echo was generated using a $h v-T_{\mathrm{DAF}}-\left(t_{\mathrm{p}}\right)-\tau-\left(3 t_{\mathrm{p}}\right)-\tau$-echo pulse sequence with $t_{\mathrm{p}}=$ $8 \mathrm{~ns}(\pi / 3)$, and $\tau_{0}=80 \mathrm{~ns}$ and $\Delta \tau=4 \mathrm{~ns}$. The echo intensity was integrated over a $100 \mathrm{~ns}$ window centered at the echo maximum. The delay between laser flash and initial microwave pulse, $T_{\mathrm{DAF}}$ was 300 ns.

\section{W-band EPR experiments}

High-field EPR measurements were performed on a home-built W-band (95 GHz/3.4 T) multipurpose EPR spectrometer 
Table 1 Kinetic parameters of $\mathrm{P}_{700}^{\cdot+}$ and $\mathrm{A}_{1}^{\cdot-}$

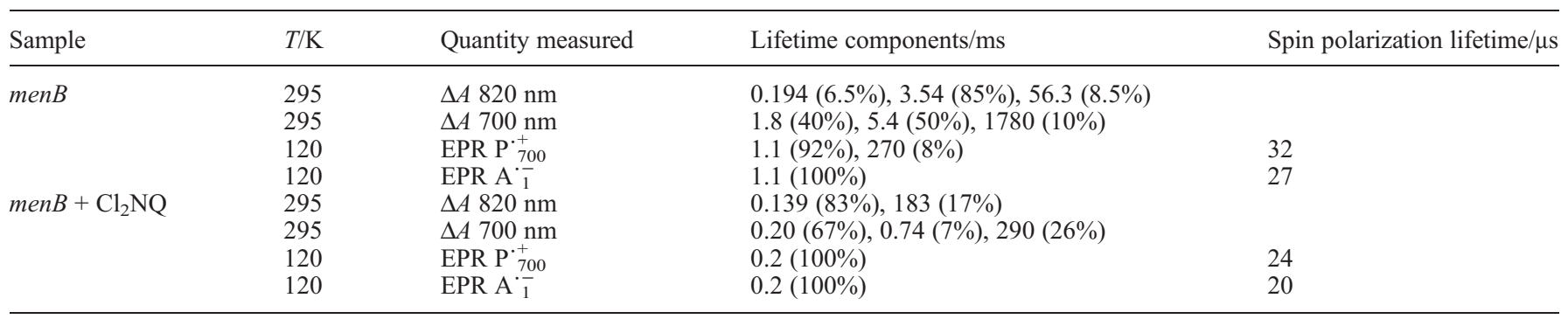
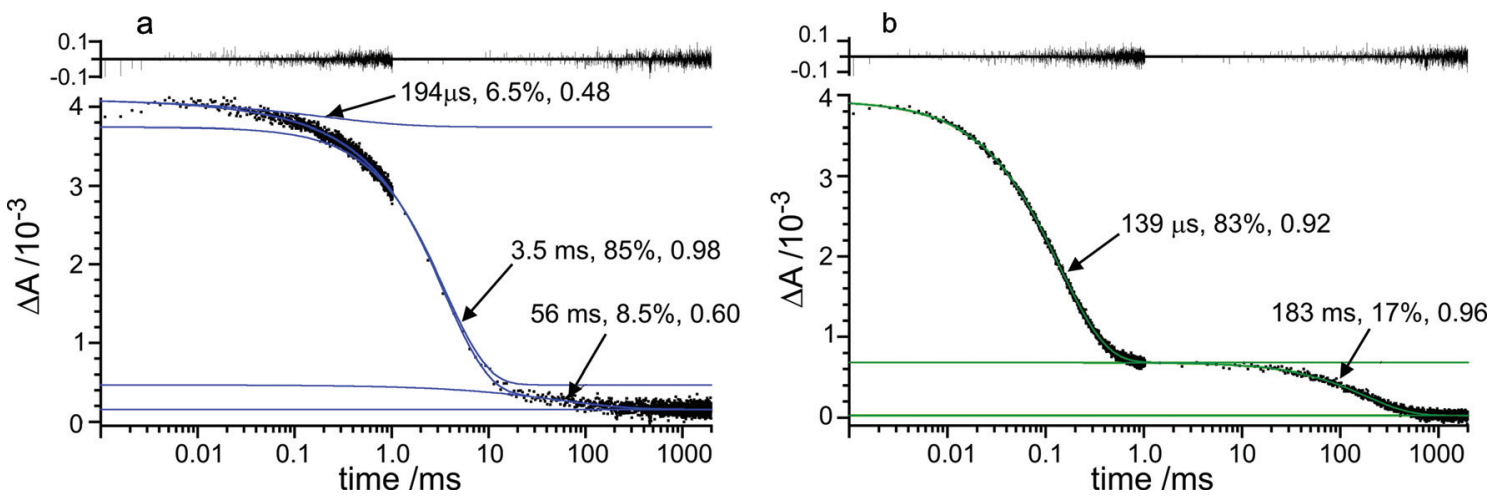

Fig. 3 Room temperature $\mathrm{P}_{700}^{+}$reduction kinetics measured at $820 \mathrm{~nm}$ in the $m e n B$ variant (a) and the $m e n B$ variant incubated with $\mathrm{Cl}_{2} \mathrm{NQ}$ (b). The experimental absorption difference data are shown in black and the blue and green curves are fits of a weighted sum of stretched exponentials to the data. The lifetimes, relative amplitudes and stretch factors of the individual kinetics components are indicated. The residuals are shown above the fit.

described previously. ${ }^{39,40}$ The sample solutions were placed in a quartz capillary (0.6 mm I.D.), transferred to the EPR probe head and, after dark adaptation at room temperature for $10 \mathrm{~min}$, cooled down to $120 \mathrm{~K}$. Light-induced electron transfer was initiated by light pulses at $532 \mathrm{~nm}$ (Nd:YAG laser, $5 \mathrm{~ns}$ pulse width, $0.5 \mathrm{~mJ}$ on the sample surface) guided to the center of the $\mathrm{TE}_{011}$ optical transmission EPR cavity through a quartz fiber of $0.8 \mathrm{~mm}$ diameter.

The recombination kinetics were obtained by recording the short-lived EPR absorption after laser flash via lock-in detection with magnetic field modulation $(30 \mathrm{kHz}, 0.1 \mathrm{mT}$ modulation amplitude). The time resolution of this detection technique was thus set to about $100 \mu \mathrm{s}$. The pulsed EPR measurements were performed using the Hahn-echo sequence $\left(t_{\mathrm{p}}\right)-\tau$ - $\left(2 t_{\mathrm{p}}\right)-\tau$-echo allowing for a time after laser flash, $T_{\mathrm{DAF}}$, in the sequence $h v-T_{\mathrm{DAF}}-\left(t_{\mathrm{p}}\right)_{x,-x}-\tau-\left(2 t_{\mathrm{p}}\right)-\tau$-echo under pulsed light illumination with repetition rate of $2 \mathrm{~Hz}$. The $t_{\mathrm{p}}$ pulse length of the $\pi / 2$ microwave (mw) pulses was generally set to $30 \mathrm{~ns}$. The quadraturedetected echo traces, $\left(s_{-y} s_{x}\right)$, were digitized and transferred to the computer for further evaluation. To obtain the in-phase fieldswept EPR spectra, the $s_{-y}$ echo response traces at $\tau=150 \mathrm{ns,}$ corresponding to the first pulse phase settings $+x$ and $-x$, were subtracted from each other, and the pure echo response, i.e., free of FID and cavity ringing signals, was integrated over the whole echo duration. The out-of-phase EPR decay profiles were evaluated from $s_{x}$ echo traces by integrating the echo responses over the time window, centered at the $s_{-y}$ echo maximum, that covers $60 \%$ of the echo intensity to optimize resolution and signal-tonoise ratio.

The single-frequency pulse dipolar EPR experiment of the RIDME type is based on measuring the out-of-phase stimulated echo signal $\left(h v-T_{\mathrm{DAF}^{-}}\left(t_{\mathrm{p}}\right)_{x,-x}-\tau-\left(t_{\mathrm{p}}\right)-T-\left(t_{\mathrm{p}}\right)-\tau-e c h o\right)$ as a function of the preparation time $\tau$. ${ }^{41}$ The fixed mixing time $T$ should be long enough to allow the longitudinal spin relaxation to flip the partner spins in the pair, but short enough to avoid a considerable reduction of the echo signal caused by the longitudinal spin relaxation of the observer spins and by the charge recombination. At $120 \mathrm{~K}$ the mean relaxation time for the radical pair was measured, by probing 2-pulse echoes vs. $T_{\mathrm{DAF}}$, to be about $30 \mu$ s. Thus, a setting of $T=20 \mu$ s satisfies the RIDME requirement. The complete RIDME data set is composed of recordings $\left(T_{\mathrm{DAF}}=400 \mathrm{~ns}, \tau_{0}=50 \mathrm{~ns}, \Delta \tau=10 \mathrm{~ns}\right)$ detected at field positions stepped through the $\mathrm{A}_{1}$ spectral region.

\section{Results}

\section{Room-temperature $\mathbf{P}_{700}^{+}$recombination kinetics}

The kinetics of charge recombination in PS I following a saturating light flash have been shown to be dependent on the midpoint potential of the quinone in the $\mathrm{A}_{1 \mathrm{~A}}$ and $\mathrm{A}_{1 \mathrm{~B}}$ sites. ${ }^{42,43}$ Hence, the back-reaction kinetics can be used to monitor the incorporation of $\mathrm{Cl}_{2} \mathrm{NQ}$ into the binding site. As displayed in Fig. 3, and summarized in Table 1, the absorbance difference traces taken in the near-IR show that the main kinetic component of the back reaction has lifetimes of $3.5 \mathrm{~ms}$ and $140 \mu \mathrm{s}$ in PS I from the men $B$ variant and the menB variant incubated with $\mathrm{Cl}_{2} \mathrm{NQ}$, respectively. The trace from the incubated sample (Fig. 3b) shows no evidence of the $3.5 \mathrm{~ms}$ decay associated with the presence of plastoquinone-9. The main kinetic components of the back reaction observed at $700 \mathrm{~nm}$ under aerobic conditions had similar lifetimes (see Table 1). Hence, we conclude that 

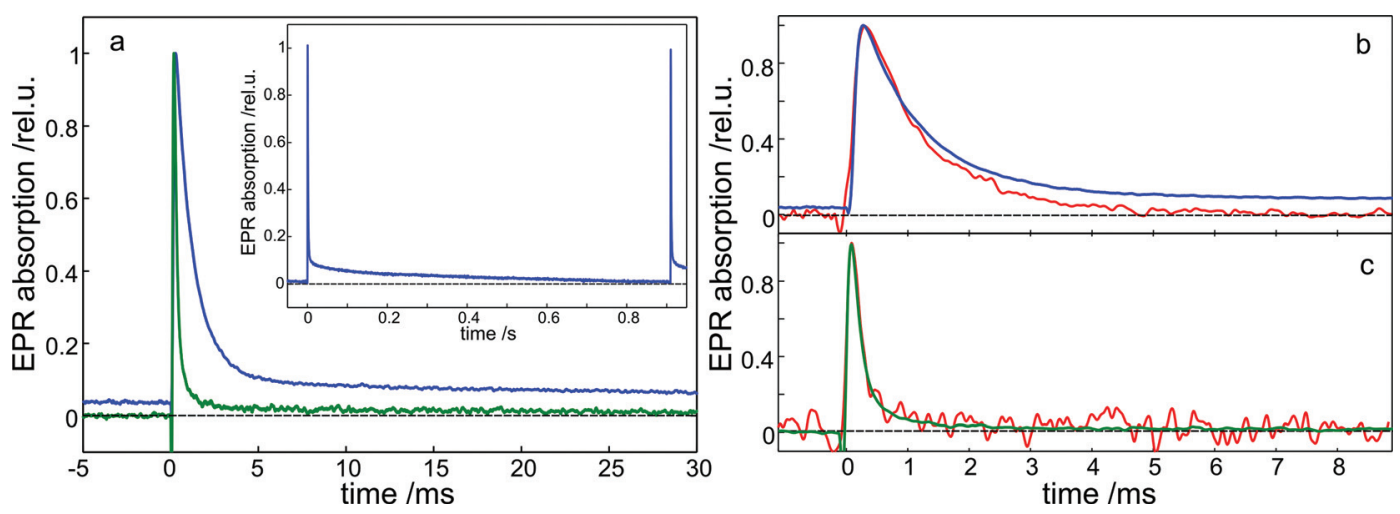

Fig. 4 Charge-recombination kinetics measured by time-resolved W-band EPR spectroscopy after pulsed laser excitation (532 nm) at $120 \mathrm{~K}$. (a) $\mathrm{P} \cdot{ }_{700}^{+}$decay in the $m e n B$ variant (blue line) and the $m e n B$ variant incubated with $\mathrm{Cl}_{2} \mathrm{NQ}$ (green line). The inset shows the decay of the $m e n B$ variant on a longer time scale. Decay traces from the $m e n B$ variant (b) and the $m e n B$ variant incubated with $\mathrm{Cl}_{2} \mathrm{NQ}_{\mathrm{C}}$ (c) taken in the $\mathrm{P}_{700}^{\cdot+}$ and $\mathrm{A}_{1}^{\cdot-}($ red line) spectral regions of the corresponding samples.

essentially complete displacement of plastoquinone- 9 by $\mathrm{Cl}_{2} \mathrm{NQ}$ has occurred in the back-reaction pathway. Both lifetimes are significantly faster than the $85 \mathrm{~ms}$ lifetime observed in the wild type. ${ }^{42}$ A positive shift of the quinone potential is expected to lead to faster back-reaction because the equilibrium between forward and back electron transfer between the quinone and $\mathrm{F}_{\mathrm{X}}$ will shift towards the quinone, resulting in a faster overall rate. ${ }^{43}$ Consistent with this expectation, the rate of the back reaction with $\mathrm{Cl}_{2} \mathrm{NQ}$ in the binding site is $\sim 30$ times faster than with plastoquinone- 9 , and $~ 900$ times faster than with phylloquinone. The small slow component is due to donation to $\mathrm{P}_{700}^{+}$by the external donor DCPIP when the transferred electron is lost to oxygen or other acceptors.

\section{Recombination kinetics at $120 \mathrm{~K}$}

At low temperature the recombination kinetics can be monitored by W-band EPR spectroscopy. At high external magnetic field, the contributions from $\mathrm{P}_{700}^{+}$and $\mathrm{A}_{1}^{--}$are spectrally well separated, due to the difference in their $g$-values, and hence their kinetics can be measured independently of one another. Previous studies ${ }^{11,22}$ suggest that at low temperature at least three fractions exist following illumination. The majority of electrons recombine from $\mathrm{A}_{1}^{--}$, a second fraction is trapped as $\mathrm{P}_{700}^{+}\left(\mathrm{F}_{\mathrm{A}} / \mathrm{F}_{\mathrm{B}}\right)^{-}$and a small amount of recombination occurs from the iron-sulfur clusters, probably from $\mathrm{F}_{\mathrm{x}}^{-}$. The relative magnitudes of the recombining fractions can be estimated from high-field EPR transient responses taken in the $\mathrm{P}_{700}^{+}$and $\mathrm{A}_{1}^{\cdot-}$ regions of the spectrum. Fig. 4 shows a comparison of such transients for the men $B$ variant and the men $B$ variant incubated with $\mathrm{Cl}_{2} \mathrm{NQ}$. The recombination lifetimes obtained from the traces are summarized in Table 1. In Fig. 4a, transients of the two samples, taken in the $\mathrm{P}_{700}^{+}+{ }^{+}$region, are compared. As expected, the lifetime of the decay from the $\mathrm{Cl}_{2} \mathrm{NQ}$ sample $(0.2 \mathrm{~ms}$, green trace $)$ is considerably shorter than that in the men $B$ sample (1.1 ms, blue trace). In addition, the men $B$ decay is clearly bi-exponential and offset from zero whereas for the $\mathrm{Cl}_{2} \mathrm{NQ}$ sample the slow component is absent and the whole signal decays to zero in less than $5 \mathrm{~ms}$. As shown in the inset of Fig. 4a, the time between consecutive laser flashes is $900 \mathrm{~ms}$, thus the offset of the curve prior to each flash represents the "stable" fraction that decays with a time longer than the repetition rate. In Fig. $4 \mathrm{~b}$ traces from the men $B$ variant taken in the $\mathrm{P}_{700}^{+}$(blue trace) and $\mathrm{A}_{1}^{--}$(red trace) regions are compared. As can be seen, the $\mathrm{A}_{1}^{--}$curve decays with the same $1.1 \mathrm{~ms}$ lifetime as the fast component of the $\mathrm{P}_{700}^{+}$trace and, thus, we can conclude that this lifetime characterizes the recombination of $\mathrm{P}_{700}^{+} \mathrm{A}_{1}^{\cdot-}$. The fact that the slow-component and offset signals are not observed in the $\mathrm{A}_{1}^{--}$region suggests that they are due to recombination from $\mathrm{F}_{\mathrm{X}}^{-}$and trapping of $\left(\mathrm{F}_{\mathrm{A}} / \mathrm{F}_{\mathrm{B}}\right)^{-}$, respectively. Since these two components are missing in the trace from the $\mathrm{Cl}_{2} \mathrm{NQ}$ sample (Fig. $4 \mathrm{c}$ ), we conclude that electron transfer past the quinone is blocked. Also shown in Table 1 are the decay times of the spin polarization of $\mathrm{P}_{700}^{+} \mathrm{A}_{1}^{--}$determined by monitoring the echo amplitude as a function of the delay time after the laser flash, $T_{\mathrm{DAF}}$. The decay of the spin polarization due to spin-lattice relaxation, $T_{1}$, is monoexponential and has the same lifetime in both the $\mathrm{P}_{700}^{+}$and $\mathrm{A}_{1}^{--}$regions of the spectrum. Following the decay of the initial spin polarization to a Boltzmann population distribution, the spin echo signal decays due to charge recombination with the same lifetimes measured using field modulation. Thus, there are no additional faster chargerecombination processes present.

\section{Accumulation of $\left(\mathrm{F}_{\mathrm{A}} / \mathrm{F}_{\mathrm{B}}\right)^{-}$at $15 \mathrm{~K}$}

The ability of the PS I samples upon illumination to transfer electrons past the quinones can also be monitored by measuring the $\mathrm{CW}$ EPR spectrum of photoaccumulated $\left(\mathrm{F}_{\mathrm{A}} / \mathrm{F}_{\mathrm{B}}\right)^{-}$at $15 \mathrm{~K}$. A comparison of these spectra for the wild type, men $B$ variant and men $B$ variant with $\mathrm{Cl}_{2} \mathrm{NQ}$ is shown in Fig. 5. In agreement with a previous repor ${ }^{27}$ the amplitude of the photoaccumulated $\left(\mathrm{F}_{\mathrm{A}} /\right.$ $\left.\mathrm{F}_{\mathrm{B}}\right)^{-}$spectrum from the wild type and menB variant is virtually the same. Note that the peak at about the free-electron $g$-value near $350 \mathrm{mT}$ is primarily from $\mathrm{P}_{700}^{+}$and is strongly saturated by the high microwave power levels needed to observe $\left(\mathrm{F}_{\mathrm{A}} / \mathrm{F}_{\mathrm{B}}\right)^{-}$. Any other light-induced organic radicals produced in the sample would also contribute in this field region. Because of the saturation and possible presence of other radical species in the sample, the intensity of this EPR peak cannot be reliably interpreted. When the men $B$ variant is incubated with a 100 -fold 


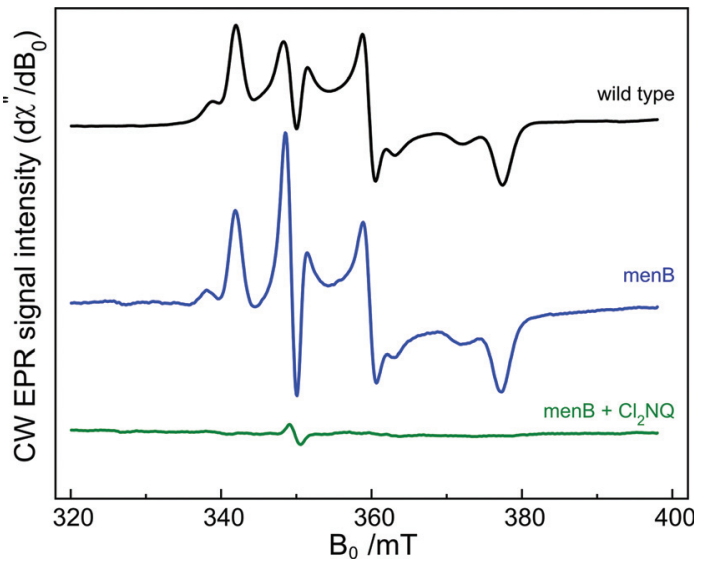

Fig. 5 Light-induced $\mathrm{X}$-band $\mathrm{CW}$ EPR spectra at $15 \mathrm{~K}$ of $\left(\mathrm{F}_{\mathrm{A}} / \mathrm{F}_{\mathrm{B}}\right)^{-}$in PS I samples frozen in the dark from the wild type (black), men $B$ variant (blue) and men $B$ variant incubated with $\mathrm{Cl}_{2} \mathrm{NQ}$ (green). In each case, a dark background spectrum, collected prior to illumination, was subtracted from the spectrum after illumination. For all three samples, the chlorophyll concentration was $2 \mathrm{mg} \mathrm{mL}^{-1}$, the modulation amplitude was $1.0 \mathrm{mT}$ and the microwave power was $1.0 \mathrm{~mW}$. No additional normalization of the spectra was performed.

excess of $\mathrm{Cl}_{2} \mathrm{NQ}$, only a weak $g \approx 2$ signal is observed and no accumulation of $\left(\mathrm{F}_{\mathrm{A}} / \mathrm{F}_{\mathrm{B}}\right)^{-}$occurs (Fig. 5, bottom). With smaller amounts of $\mathrm{Cl}_{2} \mathrm{NQ}$ (5- to 10-fold excess) a weak $\left(\mathrm{F}_{\mathrm{A}} / \mathrm{F}_{\mathrm{B}}\right)^{-}$spectrum becomes visible (data not shown) suggesting that a $100-$ fold excess is needed to fully displace plastoquinone- 9 from the $\mathrm{A}_{1}$ binding sites. Thus, consistent with the kinetic data, the lack of accumulation of $\left(\mathrm{F}_{\mathrm{A}} / \mathrm{F}_{\mathrm{B}}\right)^{-}$indicates that $\mathrm{Cl}_{2} \mathrm{NQ}$ has been incorporated and that it prevents electron transfer past the quinones.

\section{Low-temperature TREPR spectra of $\mathrm{P}_{700}^{+} \mathrm{A}_{1}^{--}$}

Fig. 6 shows a comparison of the low-temperature spin-polarized TREPR absorption-emission spectra of the radical pair $\mathrm{P}_{700}^{+} \mathrm{A}_{1}^{--}$ in PS I particles from the men $B$ variant containing plastoquinone-9 (blue spectra) and $\mathrm{Cl}_{2} \mathrm{NQ}$ (green spectra) taken at three different microwave frequencies, X-band (9 GHz, Fig. 6a), Qband (35 GHz, Fig. 6b) and W-band (95 GHz, Fig. 6c). At all three frequencies the upfield regions, which are dominated by contributions from $\mathrm{P}_{700}^{+}$, are virtually identical while the downfield regions, which arise from the quinones, differ markedly. At X-band, (Fig. 6a) the spectral width is determined to a large extent by the proton hyperfine couplings. Plastoquinone9 has three methyl groups and one methylene group with $\beta$-protons with large hyperfine couplings, ${ }^{44}$ while $\mathrm{Cl}_{2} \mathrm{NQ}$ has only ring $\alpha$-protons with small hyperfine couplings. Therefore, the X-band spectrum from the menB sample (blue) has a significantly larger width than that of the $\mathrm{Cl}_{2} \mathrm{NQ}$ sample (green). As the microwave frequency and corresponding resonance field are increased, the Zeeman energy becomes increasingly important and the quinone $g$-tensor components become better resolved at Q-band (Fig. 6b) and completely resolved at W-band (Fig. 6c). The two chlorine atoms in $\mathrm{Cl}_{2} \mathrm{NQ}$ provide a significant source of spin-orbit coupling and, thus, $\mathrm{Cl}_{2} \mathrm{NQ}$ has a larger g-anisotropy than plastoquinone- 9 . As a result, the spectral features associated
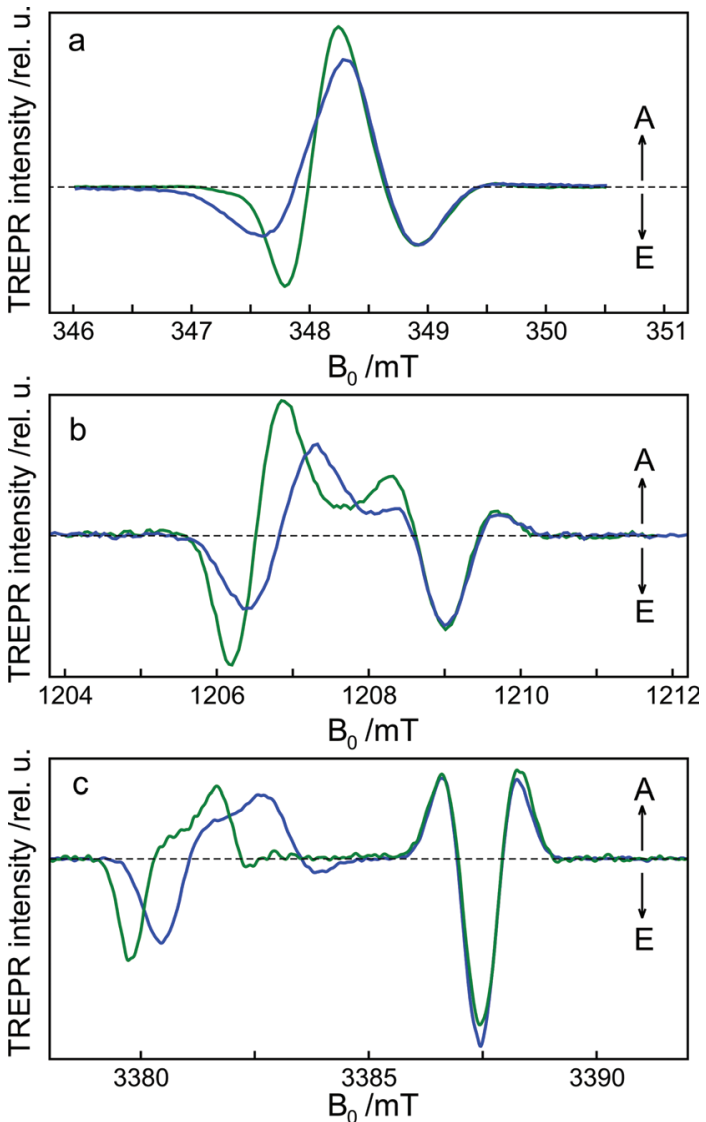

Fig. 6 Spin-polarized transient EPR spectra of the men $B$ variant (blue) and the men $B$ variant incubated with $\mathrm{Cl}_{2} \mathrm{NQ}$ (green). (a) X-band, $80 \mathrm{~K}$; (b) Q-band, $80 \mathrm{~K}$; (c) W-band, $120 \mathrm{~K}$. The X- and Q-band spectra are the direct detection transient EPR signal, while the W-band spectrum is the echo-detected spectrum. In all cases the spectrum is the difference between the signal intensity $400 \mathrm{~ns}$ after the laser flash and the intensity before the flash. The spectra are normalized in such a way that the upfield features, which are primarily due to $\mathrm{P}_{700}^{+}$, have the same amplitude for both samples.

with the $x$ - and $y$-components of the quinone $g$-tensor are shifted downfield in the Q-band and W-band spectra of the $\mathrm{Cl}_{2} \mathrm{NQ}$ sample relative to those of the men $B$ sample. Again, this confirms that $\mathrm{Cl}_{2} \mathrm{NQ}$ has been incorporated into the $\mathrm{A}_{1}$ binding site.

The polarization pattern in the $\mathrm{P}_{700}^{+}$region of the $\mathrm{W}$-band spectrum is sensitive to the orientation of the dipolar coupling vector relative to the principal axes of the $\mathrm{P}_{700}^{+} \mathrm{A}_{1}^{--}$g-tensor. ${ }^{45}$ Because this orientation is different in the radical pairs $\mathrm{P}_{700}^{+} \mathrm{A}_{1 \mathrm{~A}}^{-}$and $\mathrm{P}_{700}^{+} \mathrm{A}_{1 \mathrm{~B}}^{-}$of PS I, their high-field EPR spectra differ in the $\mathrm{P}_{700}^{+}$region, as has been demonstrated using prereduced PS I samples. ${ }^{18}$ The fact that this region is virtually identical in the men $B$, men $B$ plus $\mathrm{Cl}_{2} \mathrm{NQ}$ and wild-type (not shown) samples indicates that there is no additional contribution from the B-branch radical pair when electron transfer past the quinone is blocked in the $\mathrm{Cl}_{2} \mathrm{NQ}$ sample. Simulation of the quinone region of the W-band spectra reveals that the spectrum of the $\mathrm{Cl}_{2} \mathrm{NQ}$ sample contains a minor contribution $(12 \pm 3 \%)$ from plastoquinone-9 in the $\mathrm{A}_{1}$ site. Since the $\mathrm{Cl}_{2} \mathrm{NQ}$ samples for all of the experiments reported here were prepared in the 
Table 2 Magnetic and geometric parameters evaluated from the W-band time-resolved EPR spectra and W-band out-of-phase ESEEM of the spincorrelated radical pair $\mathrm{P}_{700}^{\cdot+} \mathrm{A}_{1}^{\cdot-}$

\begin{tabular}{llllll}
\hline & $\mathrm{A}_{1}^{-} g$-tensor $\left(g_{x}, g_{y}\right)^{a}$ & Linewidths $\Delta B_{1 / 2} / \mathrm{mT}(x, y)^{a}$ & $\operatorname{Tr}_{\mathrm{Ay} / x}$ & Dipolar frequency $v_{\perp} / \mathrm{MHz}$ & Distance $r_{\mathrm{AP}} / \mathrm{nm}$ \\
\hline Phylloquinone (perdeuterated WT) & $2.00623,2.00507$ & $0.34,0.40$ & $-0.56 \pm 0.05$ & $2.94 \pm 0.05$ & $2.61 \pm 0.02$ \\
Plastoquinone-9 (menB variant) & $2.00680,2.00519$ & $0.86,1.09$ & $-0.61 \pm 0.05$ & $3.00 \pm 0.05$ & $2.59 \pm 0.02$ \\
$\mathrm{Cl}_{2}$ NQ (menB variant) & $2.00722,2.00590$ & $0.54,0.65$ & $-0.58 \pm 0.05$ & $2.92 \pm 0.05$ & $2.61 \pm 0.02$
\end{tabular}

${ }^{a} g_{z}$ value and the corresponding linewidth could not be determined due to overlap of the $\mathrm{A}_{1}^{\cdot-}$ and $\mathrm{P}_{700}^{\cdot+}$ spectral contributions.
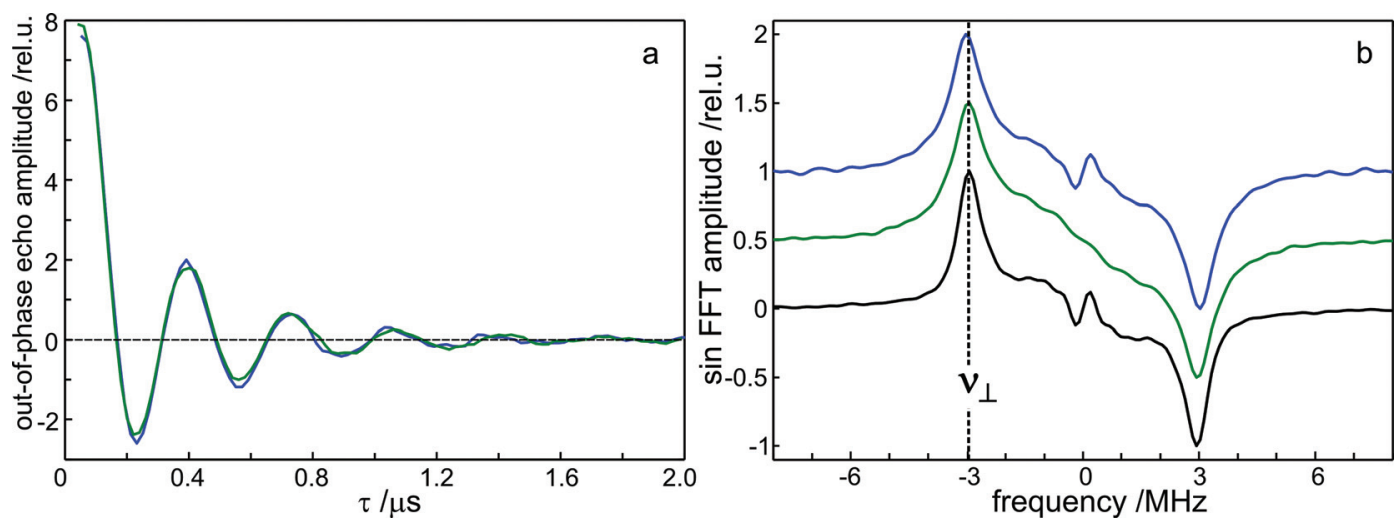

Fig. 7 W-band out-of-phase ESEEM measurements of the men $B$ variant and men $B$ incubated with $\mathrm{Cl}_{2} \mathrm{NQ}$ at $120 \mathrm{~K}$. (a) Out-of-phase ESEEM traces taken at the $\mathrm{P}_{700}^{+}$signal maximum for the $m e n B$ variant (blue) and $\mathrm{Cl}_{2} \mathrm{NQ}$ sample (green). The delay-after-flash time, TDAF, was set to $400 \mathrm{~ns}$ and a laser repetition rate of $10 \mathrm{~Hz}$ was used. The signals are scaled to the maximum amplitude. (b) Sine Fourier transform amplitudes of the ESEEM decays for the men $B$ variant (blue) and $\mathrm{Cl}_{2} \mathrm{NQ}$ sample (green). For comparison, the corresponding Fourier transform for perdeuterated wild type PS I is also shown (black line). The intensities around zero frequency are due to residuals after the baseline correction.

same way, this value can be taken as representative of the degree of incorporation for all of the samples. With this contribution taken into account, the simulations yield the parameters given in Table 2. Although the spectra do not yield the absolute orientation of the quinone, they depend on the geometric parameter, $\operatorname{Tr}_{\mathrm{A} y / x}$ determined by the ratio of the projections of the dipolarcoupling vector onto the principal $y$ - and $x$-axes of the $\mathrm{A}^{\cdot-} g$ tensor: ${ }^{41}$

$$
\operatorname{Tr}_{\mathrm{A} y / x}=\frac{\left(1-3 \sin ^{2} \eta_{\mathrm{A}} \sin ^{2} \phi_{\mathrm{A}}\right)}{\left(1-3 \sin ^{2} \eta_{\mathrm{A}} \cos ^{2} \phi_{\mathrm{A}}\right)}
$$

The polar angles $\eta_{\mathrm{A}}$ and $\phi_{\mathrm{A}}$ are the inclination and azimuth angles determining the direction of the electron-electron interspin vector $\mathbf{r}_{\mathrm{AP}}$ in the $g$-tensor frame of $\mathrm{A}_{1}^{\cdot}$. As can be seen from Table 2, the value of this geometric parameter is the same, within experimental error, for phylloquinone, plastoquinone-9 and $\mathrm{Cl}_{2} \mathrm{NQ}$, which suggests that all three quinones are bound in their PS I binding sites in the same orientation.

\section{Distance between $\mathrm{P}_{700}^{++}$and $\mathrm{A}_{\mathbf{1}}^{\cdot-}$}

The spin density distribution in $\mathrm{P}_{700}^{\cdot+}$ (a Chla/Chl $a^{\prime}$ dimer) is highly asymmetric and resides primarily on the eC-B1 chlorophyll. $^{46-48}$ Because of this asymmetry, the distances between radical centers in $\mathrm{P}_{700}^{\cdot+} \mathrm{A}_{1 \mathrm{~A}}{ }^{\cdot-}$ and $\mathrm{P}_{700}^{++} \mathrm{A}_{1 \mathrm{~B}}^{\cdot-}$ are different. The dipolar coupling between $\mathrm{P}_{700}^{+}$and $\mathrm{A}_{1}^{\cdot-}$ depends on this distance and, therefore, can be used to deduce the contributions of the two possible radical pairs to the EPR signals. The electron-electron spin-spin coupling in the spin-polarized transient radical pair $\mathrm{P}_{700}^{++} \mathrm{A}_{1}^{\cdot-}$ is measured most conveniently using the out-of-phase ESEEM technique. ${ }^{49,50}$ In PS I particles from the wild type ${ }^{51-53}$ and $m e n B$ variant ${ }^{28}$ such measurements yield distances consistent with the A-branch charge-separated radical pair. Fig. 7 shows a comparison of W-band out-of-phase ESEEM data from the perdeuterated wild-type, men $B$ variant and menB incubated with $\mathrm{Cl}_{2} \mathrm{NQ}$ taken at the $\mathrm{P}_{700}^{\cdot+}$ spectral region. In this region the modulation frequency observed in the out-of-phase detected echo decay corresponds to the perpendicular dipolar coupling frequency, $v_{\perp}$. This is because the dipolar vectors directed from $\mathrm{P}_{700}^{+}$to the semiquinones $\mathrm{A}_{1 \mathrm{~A}}{ }^{\cdot-}$ and $\mathrm{A}^{\cdot-} \mathrm{B}$ are oriented almost perpendicularly to the $z$-axes of both quinones. ${ }^{18}$ As can be seen, the echo-modulation curves (Fig. 7a) from the men $B$ variant and menB incubated with $\mathrm{Cl}_{2} \mathrm{NQ}$ samples are almost identical. The sine Fourier transforms of the modulation curves (Fig. 7b) show single peaks at $v_{\perp}$. From the calibration relation in the point-dipole approximation $v_{\perp}=52.04\left(r_{0} /\right.$ $\left.r_{\mathrm{AP}}\right)^{3} / \mathrm{MHz}\left(r_{0}=1 \mathrm{~nm}\right)^{41}$ the distance between the radicals can be determined from the peak positions. The contribution of the exchange coupling $J \leq 0.03 \mathrm{MHz}$ to the perpendicular dipolar coupling frequency, $v_{\perp}$, can be safely ignored in the distance calculation from the obtained dipolar frequency. ${ }^{53}$ The distances obtained for the three samples are given in Table 2, and within experimental error they are all identical. Any possible contribution from the B-branch radical pair or distribution of distances would be easily recognized as differences in the lineshapes of the peaks in the Fourier transforms of the modulation curves. ${ }^{22}$ 

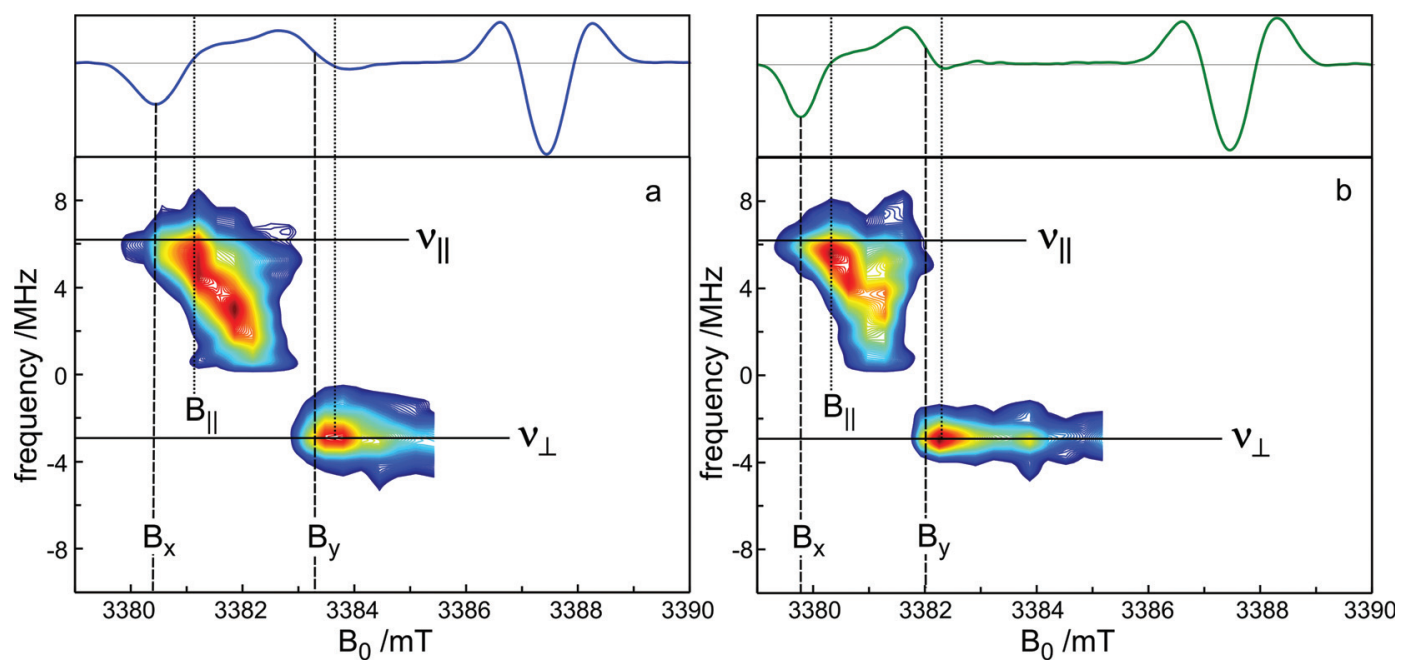

Fig. 8 W-band dipolar out-of-phase RIDME spectra of the radical pair $\mathrm{P}_{700}^{+} \mathrm{A}_{1}^{\cdot-}$ at $120 \mathrm{~K}$ in $m e n B$ variant (a) and $m e n B$ incubated with $\mathrm{Cl}_{2} \mathrm{NQ}(\mathrm{b})$. The data were collected with a long mixing period of the stimulated spin-echo pulse sequence of $T=20 \mu \mathrm{s}$, and the delay after the laser flash $\mathrm{T}_{\mathrm{DAF}}=$ $200 \mathrm{~ns}$. The contour plot shows the positive sine Fourier amplitudes of the RIDME traces taken over the spectral region dominated by the $g_{x}$ and $g_{y}$ tensor components of the $\mathrm{A}_{1}^{\cdot-}$ radical. The spin-polarized TREPR spectrum of $\mathrm{P}_{700}^{+} \mathrm{A}_{1}^{\cdot-}$ is displayed above the contour plot to show the corresponding spectral positions. For additional information, see text.

However, within experimental error, all three samples have the same peak shapes as can be seen in Fig. 7b.

\section{Relaxation-induced dipolar modulation enhancement (RIDME) experiments}

The orientation and distance of the radical-pair partners can be determined together by combining the spectral resolution of high-field EPR with spin-echo modulation. ${ }^{41,54}$ In the RIDME experiment the echo amplitude from one of the radical-pair partners is modulated by the electron spin-spin coupling due to relaxation induced-spin flips of the partner radical. By plotting the Fourier-transform amplitudes of the modulations of the spinecho decay trace against field position, the dipolar coupling is correlated with the $g$-tensor components of the radicals, thus allowing the distance between them and the relative orientation of the dipolar coupling vector and the $g$-tensor axes to be deduced. Fig. 8 shows a comparison of such two-dimensional plots for the men $B$ variant and the $\mathrm{Cl}_{2} \mathrm{NQ}$ sample taken over the $g_{x}-g_{y}$ quinone region of the spectrum. The spectral positions corresponding to quinone $g_{x}$ and $g_{y}$ resonance fields, $B_{x}$ and $B_{y}$ are indicated by dashed lines. The solid horizontal lines indicate the frequencies associated with the parallel, $v_{\|}$, and perpendicular, $v_{\perp}$, components of the dipolar coupling, and the vertical dotted lines indicate the corresponding spectral positions at which the maximum modulation amplitude occurs. From the plot it is immediately apparent that the principal components of the dipolar coupling are identical in the two samples, and that they map onto the $g$-tensor axes in the same way, with the parallel component close to $g_{x}$ and the visible perpendicular component close to $g_{y}$ Again this demonstrates that the position and orientation of the quinone is the same in both samples. ${ }^{54}$ The values of the dipolar coupling are identical to those observed in the wild type so that we conclude that only the A-branch radical pair is observed.

\section{TREPR spectra of the $r u b A / m e n B$ double mutant}

All of the above data indicate that when $\mathrm{Cl}_{2} \mathrm{NQ}$ is incorporated into PS I from the men $B$ variant, electron transfer past the quinone is blocked at low temperature and that only signal contributions associated with the A-branch radical pair are observed. This suggests that the electron transfer is unidirectional in the Abranch. However, a scenario in which a fraction of the electron transfer would occur in the B-branch and proceed rapidly from $A_{1 B}$ to $A_{1 A}$ via $F_{x}$ would give similar results. Such a scenario is quite unlikely for several reasons. First, it would require that only the A-branch quinone is exchanged since fast electron transfer from $\mathrm{Cl}_{2} \mathrm{NQ}$ to $\mathrm{F}_{\mathrm{X}}$ is energetically not feasible. Second, it is well known ${ }^{55}$ that electron transfer beyond $A_{1}$ results in net polarization of $\mathrm{P}_{700}^{+}$and that this polarization has a characteristic dependence on the magnetic field/microwave frequency. ${ }^{56}$ Net polarization of $\mathrm{P}_{700}^{+}$is not observed in any of the TREPR data. Nonetheless, we can test for the possibility of B-branch electron transfer using the rubA/menB double mutant. ${ }^{37}$ This mutant is unable to synthesize phylloquinone (plastoquinone-9 is incorporated instead) and to assemble the iron-sulfur clusters. Thus, electron transfer past the quinones cannot occur. In principle, direct electron transfer between the quinones could occur, however using Dutton's ruler ${ }^{57}$ as an estimate of the distance dependence of the electron transfer rate due to the electronic coupling, we obtain a minimum lifetime for inter-quinone electron transfer on the order of $1 \mu \mathrm{s}$ from the edge-to-edge distance between the two quinones $(\sim 15 \AA)$. Thus, this electron-transfer step should be slow enough to be detectable by EPR. Fig. 9 shows a comparison of the X-band TREPR data of the men $B$ and $m e n B / r u b A$ variants before and after incubation with $\mathrm{Cl}_{2} \mathrm{NQ}$. The spin-polarized spectra of the two variants are identical with both plastoquinone-9 (Fig. 9a, top) and $\mathrm{Cl}_{2} \mathrm{NQ}$ (Fig. 9a, bottom) in the binding site. The corresponding sine Fourier transforms of the out-of-phase ESEEM curves are shown in Fig. 9b. In all four samples the dipolar frequency is $v_{\perp}=2.95 \mathrm{MHz}$ as indicated by 

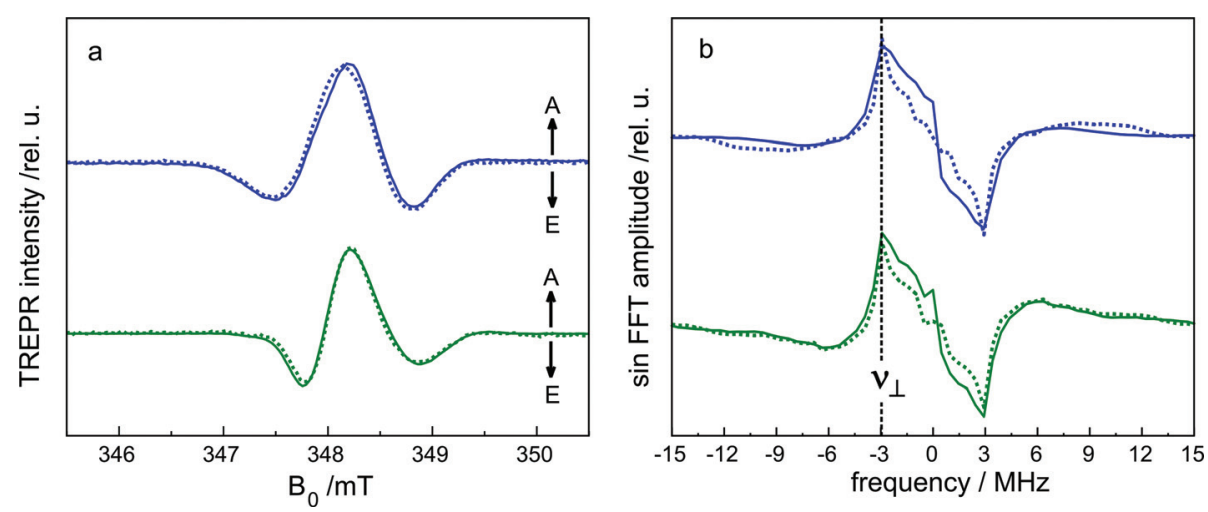

Fig. 9 Comparison of X-band TREPR data from the menB variant and menB/rubA variant of PS I. (a) Transient EPR spectra $600 \mathrm{~ns}$ after the laser flash. (b) Sine Fourier transforms of the out-of-phase ESEEM curves. The men $B$ variant data are plotted as solid lines and the data from the men $B /$ $r u b A$ variant are shown as dashed lines. The blue traces are PS I containing plastoquinone- 9 and the green traces are samples incubated with $\mathrm{Cl}_{2} \mathrm{NQ}$.

the dashed line in Fig. 9b. Thus, we conclude that in all of these samples the electron transfer is strongly biased towards the Abranch at low temperature.

\section{Discussion}

All of the above data indicate that when $\mathrm{Cl}_{2} \mathrm{NQ}$ is incorporated into PS I, electron transfer past the quinones does not occur in frozen solution. Thus, incorporation of a high-potential quinone provides an alternative to removal or reduction of $F_{X}$ for blocking forward transfer. Under these conditions we find that within the detection limits of TREPR there is no evidence for involvement of the B-branch at low temperature. This is in agreement with high-field W-band EPR spectroscopy studies of the rubA variant $^{23,58}$ and the point mutants M688 $\mathrm{N}_{\mathrm{PsaA}}$ and $\mathrm{M} 668 \mathrm{~N}_{\mathrm{PsaB}},{ }^{22}$ which show that in dark-adapted samples without pre-reduction of the iron-sulfur clusters, electron transport in cyanobacterial PS I is strongly biased towards the A-branch of cofactors below the glass-transition temperature.

In contrast, many studies in which the iron-sulfur clusters are reduced do show evidence for low-temperature B-branch electron transfer. ${ }^{4,13,16-21}$ The origin of the different results for the two types of samples is not immediately apparent but it suggests that the directionality of electron transfer in PS I is easily influenced. Since the spin-polarized EPR spectra show that $\mathrm{Cl}_{2} \mathrm{NQ}$ binds to the $\mathrm{A}_{1 \mathrm{~A}}$ site in the same position and orientation as phylloquinone, it is unlikely that exchanging the quinone has any significant effect on the directionality, which is determined by the midpoint potentials and binding of the co-factors involved in the initial charge separation. On the other hand, according to electrostatic calculations, the free energy difference between midpoint potentials of $\mathrm{A}_{1 \mathrm{~A}}$ and $\mathrm{F}_{\mathrm{X}}$ was estimated to be $+10 \mathrm{mV}^{35}$ More recent a semi-continuum electrostatic approach using two dielectric constants and substantial heterogeneity of the static dielectric constant gave the value of $\sim-80 \mathrm{mV} .{ }^{36}$ Modeling of the forward and backward electron transfer reactions in PS I yielded an energy gap between $A_{1 A}$ and $F_{X}$ of -50 to $-80 \mathrm{mV}^{59}$ Therefore it is possible that under the experimental conditions used in some previous publications, ${ }^{18,20}$ the prereduction of $\mathrm{F}_{\mathrm{X}}$ in the presence of dithionite under illumination at low temperature is accompanied by at least partial reduction of $\mathrm{A}_{1 \mathrm{~A}}$.
Indeed, it is known that because the midpoint potentials of $\mathrm{A}_{1 \mathrm{~A}}$ and $A_{1 B}$ are different, addition of dithionite and illumination leads initially to accumulation of $\mathrm{A}_{1 \mathrm{~A}}^{-}$and that $\mathrm{A}_{1 \mathrm{~B}}$ is reduced only after prolonged illumination and double reduction of $\mathrm{A}_{1 \mathrm{~A}} \cdot{ }^{16}$ Therefore the accumulation of charges on the acceptor side of the complex under reducing conditions changes the electrostatic environment and should affect $\mathrm{A}_{0 \mathrm{~A}}$ and $\mathrm{A}_{0 \mathrm{~B}}$ differently. We note also that the iron-sulfur clusters $\mathrm{F}_{\mathrm{A}}$ and $\mathrm{F}_{\mathrm{B}}$ are arranged asymmetrically with respect to the two branches and could also lead to a difference in the electrostatic effects in the two branches. The size of the overall influence of charges on the acceptor side of PS I is difficult to determine accurately, but using the calculated contributions from local charges to the midpoint potentials of the cofactors in native PS $\mathrm{I}^{35,36}$ as a guide, a shift of $10-100 \mathrm{mV}$ in the midpoint potential of $\mathrm{A}_{0 \mathrm{~A}}$ can be expected, particularly if $\mathrm{A}_{1 \mathrm{~A}}$ becomes reduced. It has been shown that disruption of the $\mathrm{H}-$ bond between tyrosine $\mathrm{Y} 696_{\mathrm{PsaA}}$ and the $\mathrm{A}_{0}$ chlorophyll eC-A3 (or between Y676 ${ }_{\mathrm{PsaB}}$ and chlorophyll eC-B3) leads to redirection of electrons into the B-branch (or A-branch) ${ }^{25}$ Thus, it is plausible that the shift in the midpoint potential due to the additional charges introduced when the acceptor side of PS I is reduced, could redirect electrons into the B-branch.

The data presented here also allow conclusions to be drawn about the origin of the different fractions observed at low temperature and the relative midpoint potentials of $\mathrm{A}_{1 \mathrm{~A}}$ and $\mathrm{A}_{1 \mathrm{~B}}$. Because electron transfer from $A_{1 B}$ to $F_{X}$ is known to be nearly activationless, while the $A_{1 A}$ to $F_{X}$ step is strongly activated, ${ }^{12}$ it has been postulated that the irreversible fraction results from electron transfer in the B-branch. The strong bias towards the Abranch suggests that if trapping does occur via B-branch electron transfer, the single-flash quantum yield of stable $\left(\mathrm{F}_{\mathrm{A}} / \mathrm{F}_{\mathrm{B}}\right)^{-}$would be very low. Moreover, the observation of back reaction from the iron-sulfur clusters at low temperature shows that electron transfer beyond the quinones does not necessarily lead to trapping and suggests that the two fractions may be the result of heterogeneity in either forward or reverse electron transfer between $F_{X}$ and $\mathrm{F}_{\mathrm{A}}$. Incorporation of quinones with potentials higher than that of phylloquinone can also be used to make a rough estimate of the difference in midpoint potential between $\mathrm{A}_{1 \mathrm{~A}}$ and $\mathrm{A}_{1 \mathrm{~B}}$. In DMF, the difference in redox potential between phylloquinone and plastoquinone- 9 and between phylloquinone and $\mathrm{Cl}_{2} \mathrm{NQ}$ are $96 \mathrm{mV}$ and $416 \mathrm{mV}$, respectively (Fig. 2). If these differences 
also apply to the protein-bound quinones and we assume that reduction of $\mathrm{F}_{\mathrm{A}} / \mathrm{F}_{\mathrm{B}}$ is the result of a small amount of $\mathrm{B}$-branch electron transfer, then the midpoint potential of $\mathrm{A}_{1 \mathrm{~B}}$ would be at least $96 \mathrm{mV}$ but less than $416 \mathrm{mV}$ more negative than that of $\mathrm{A}_{1 \mathrm{~A}}$ since an $\left(\mathrm{F}_{\mathrm{A}} / \mathrm{F}_{\mathrm{B}}\right)^{-}$spectrum is observed in the men $B$ variant but not for the $\mathrm{Cl}_{2} \mathrm{NQ}$ containing sample (Fig. 5). This estimate is in line with the differences of $155 \mathrm{mV}$ and $173 \mathrm{mV}$ calculated by Ishikita et al. ${ }^{35}$ and Ptushenko et al., ${ }^{36}$ respectively, but does not agree with the value of $25 \mathrm{mV}$ estimated by Santabarbara et al. ${ }^{34}$

Together, the observation of highly asymmetric electron transfer at low temperature, and the optical and EPR data indicating bidirectional electron transfer at room temperature, suggest that the relative use of the two branches in PS I is temperature dependent. This is consistent with the observation by Agalarov and Brettel $^{12}$ that the relative amplitude of the kinetic phase associated with electron transfer from $\mathrm{A}_{1 \mathrm{~B}}^{-}$to $\mathrm{F}_{\mathrm{X}}$ becomes smaller with decreasing temperature and could not be observed reliably below $223 \mathrm{~K}$. The fact that we see no evidence for B-branch electron transfer at low temperature in the $\mathrm{Cl}_{2} \mathrm{NQ}$ sample suggests that the difficulty observing the fast phase below $223 \mathrm{~K}$ is due to the loss of electron-transfer activity in the B-branch. If this is the case, then changes in the shape of the spin-polarized EPR spectra of $\mathrm{P}_{700}^{+} \mathrm{A}_{1}^{\cdot-}$ can be expected at room temperature in the $\mathrm{Cl}_{2} \mathrm{NQ}$ sample as the contribution from the B-branch radical pair $\mathrm{P}_{700}^{+} \mathrm{A}_{1 \mathrm{~B}}^{-}$becomes appreciable. Indeed, such differences are observed in the spectra from the rubA mutant. ${ }^{23}$ However, because the iron-clusters are absent it is difficult to exclude the possibility of structural changes at high temperature. This problem should not occur in the $\mathrm{Cl}_{2} \mathrm{NQ}$ sample, and investigations of the spin-polarized EPR spectra and kinetics at room temperature are in progress.

\section{Acknowledgements}

This work was supported by grants from the Natural Sciences and Engineering Research Council, Canada to A.v.d.E., from the National Science Foundation (MCB-1021725) to J.H.G., from the Russian Foundation for Basic Research (RFBR, 09-0401657), and from the Russian Ministry for Education and Science (16.512.12.2010) to A. Yu. S., from DFG-RFBR Cooperation Project 11-04-91330_NNIO) to A. Yu. S and K.M, and from the Max Planck Society to A.S. and K.M. We thank Mike Gorka for providing membrane fragments of the rubA/ $m e n B$ variant.

\section{References}

1 M. G. Müller, C. Slavov, R. Luthra, K. E. Redding and A. R. Holzwarth, Proc. Natl. Acad. Sci. U. S. A., 2010, 107, 4123-4128.

2 I. V. Shelaev, F. E. Gostev, M. D. Mamedov, O. M. Sarkisov, V. A. Nadtochenko, V. A. Shuvalov and A. Y. Semenov, Biochim. Biophys. Acta, Bioenerg., 2010, 1797, 1410-1420.

3 B. Boudreaux, F. MacMillan, C. Teutloff, R. Agalarov, F. Gu, S. Grimaldi, R. Bittl, K. Brettel and K. Redding, J. Biol. Chem., 2001, 276, 37299-37306.

4 W. V. Fairclough, A. Forsyth, M. C. Evans, S. E. Rigby, S. Purton and P. Heathcote, Biochim. Biophys. Acta, Bioenerg., 2003, 1606, 43-55.

5 M. Guergova-Kuras, B. Boudreaux, A. Joliot, P. Joliot and K. Redding, Proc. Natl. Acad. Sci. U. S. A., 2001, 98, 4437-4442.
6 W. Xu, P. Chitnis, A. Valieva, A. van der Est, Y. N. Pushkar, M. Krzystyniak, C. Teutloff, S. G. Zech, R. Bittl, D. Stehlik, B. Zybailov, G. Shen and J. H. Golbeck, J. Biol. Chem., 2003, 278, 27864-27875.

7 W. Xu, P. R. Chitnis, A. Valieva, A. van der Est, K. Brettel, M. Guergova-Kuras, Y. N. Pushkar, S. G. Zech, D. Stehlik, G. Shen, B. Zybailov and J. H. Golbeck, J. Biol. Chem., 2003, 278, 27876-27887.

8 K. Redding and A. van der Est, in Photosystem I the Light-Driven Plastocyanin:Ferrodoxin Oxidoreductase, ed. J. Golbeck, Springer, Dordrecht, The Netherlands, 2006, pp. 413-437.

9 K. Redding, S. Santabarbara, A. Jasaitis, M. Byrdin, F. F. Gu and F. Rappaport, Photochem. Photobiol., 2008, 84, 1381-1387.

10 K. Brettel, Biochim. Biophys. Acta, Bioenerg., 1997, 1318, 322-373.

11 E. Schlodder, K. Falkenberg, M. Gergeleit and K. Brettel, Biochemistry, 1998, 37, 9466-9476.

12 R. Agalarov and K. Brettel, Biochim. Biophys. Acta, Bioenerg., 2003, 1604, 7-12.

13 N. Srinivasan, I. Karyagina, R. Bittl, A. van der Est and J. H. Golbeck, Biochemistry, 2009, 48, 3315-3324.

14 R. O. Cohen, G. Shen, J. H. Golbeck, W. Xu, P. R. Chitnis, A. I. Valieva, A. van der Est, Y. Pushkar and D. Stehlik, Biochemistry, 2004, 43, 47414754.

15 A. van der Est, S. Chirico, I. Karyagina, R. Cohen, G. Shen and J. H. Golbeck, Appl. Magn. Reson., 2010, 37, 103-121.

16 P. Heathcote, S. E. J. Rigby, I. P. Muhiuddin, M. C. W. Evans and S. Purton, Biochim. Biophys. Acta, Bioenerg., 2002, 1556, 13-20.

17 P. Heathcote, S. Santabarbara, I. Kuprov, W. V. Fairclough, S. Purton, P. J. Hore and M. C. W. Evans, Biochemistry, 2005, 44, 2119-2128.

18 O. G. Poluektov, S. V. Paschenko, L. M. Utschig, K. V. Lakshmi and M. C. Thurnauer, J. Am. Chem. Soc., 2005, 127, 11910-11911.

19 S. Santabarbara, K. Ali, P. Heathcote, M. C. W. Evans and S. Purton, Biochim. Biophys. Acta, Bioenerg., 2006, 1757, 1623-1633.

20 S. Santabarbara, I. Kuprov, P. J. Hore, A. Casal, P. Heathcote and M. C. W. Evans, Biochemistry, 2006, 45, 7389-7403.

21 S. Santabarbara, I. Kuprov, O. Poluektov, A. Casal, C. A. Russell, S. Purton and M. C. W. Evans, J. Phys. Chem. B, 2010, 114, 1515815171.

22 A. Savitsky, O. Gopta, M. Mamedov, J. H. Golbeck, A. Tikhonov, K. Möbius and A. Semenov, Appl. Magn. Reson., 2009, 37, 85-102.

23 G. Z. Shen, M. L. Antonkine, A. van der Est, I. R. Vassiliev, K. Brettel, R. Bittl, S. G. Zech, J. D. Zhao, D. Stehlik, D. A. Bryant and J. H. Golbeck, J. Biol. Chem., 2002, 277, 20355-20366.

24 G. Z. Shen, J. D. Zhao, S. K. Reimer, M. L. Antonkine, Q. Cai, S. M. Weiland, J. H. Golbeck and D. A. Bryant, J. Biol. Chem., 2002, 277, 20343-20354.

25 Y. J. Li, A. van der Est, M. G. Lucas, V. M. Ramesh, F. F. Gu, A. Petrenko, S. Lin, A. N. Webber, F. Rappaport and K. Redding, Proc. Natl. Acad. Sci. U. S. A., 2006, 103, 2144-2149.

26 J. H. Golbeck, R. Agalarov, M. Byrdin, F. Rappaport, G. Z. Shen, D. A. Bryant and A. van der Est, Photochem. Photobiol., 2008, 84, $1371-1380$.

27 T. W. Johnson, G. Z. Shen, B. Zybailov, D. Kolling, R. Reategui, S. Beauparlant, I. R. Vassiliev, D. A. Bryant, A. D. Jones, J. H. Golbeck and P. R. Chitnis, J. Biol. Chem., 2000, 275, 8523-8530.

28 B. Zybailov, A. van der Est, S. G. Zech, C. Teutloff, T. W. Johnson, G. Z. Shen, R. Bittl, D. Stehlik, P. R. Chitnis and J. H. Golbeck, J. Biol. Chem., 2000, 275, 8531-8539.

29 A. van der Est, Y. Pushkar, I. Karyagina, B. Fonovic, T. Dudding, J. Niklas, W. Lubitz and J. H. Golbeck, Appl. Magn. Reson., 2010, 37, 65-83.

30 Y. N. Pushkar, S. G. Zech, D. Stehlik, S. Brown, A. van der Est and H. Zimmermann, J. Phys. Chem. B, 2002, 106, 12052-12058.

31 Y. N. Pushkar, D. Stehlik, M. van Gastel and W. Lubitz, J. Mol. Struct., 2004, 700, 233-241.

32 Y. N. Pushkar, J. H. Golbeck, D. Stehlik and H. Zimmermann, J. Phys. Chem. B, 2004, 108, 9439-9448.

33 Y. N. Pushkar, O. Ayzatulin and D. Stehlik, Appl. Magn. Reson., 2005, 28, 195-211.

34 S. Santabarbara, P. Heathcote and M. C. W. Evans, Biochim. Biophys. Acta, 2005, 1708, 283-310.

35 H. Ishikita and E. W. Knapp, J. Biol. Chem., 2003, 278, 52002-52011.

36 V. V. Ptushenko, D. A. Cherepanov, L. I. Krishtalik and A. Y. Semenov, Photosyn. Res., 2008, 97, 55-74.

37 Y. Sakuragi, B. Zybailov, G. Z. Shen, D. A. Bryant, J. H. Golbeck, B. A. Diner, I. Karyagina, Y. Pushkar and D. Stehlik, J. Biol. Chem., 2005, 280, 12371-12381. 
38 N. Srinivasan, S. Santabarbara, F. Rappaport, D. Carbonera, K. Redding, A. van der Est and J. H. Golbeck, J. Phys. Chem. B, 2011, 115, 1751-1759.

39 K. Möbius and A. Savitsky, High-Field EPR Spectroscopy on Proteins and their Model Systems: Characterization of Transient Paramagnetic States, RSC Publishing, London, 2009.

40 K. Möbius, A. Savitsky, A. Schnegg, M. Plato and M. Fuchs, Phys. Chem. Chem. Phys., 2005, 7, 19-42.

41 A. Savitsky, A. A. Dubinskii, M. Flores, W. Lubitz and K. Möbius, J. Phys. Chem. B, 2007, 111, 6245-6262.

42 A. Y. Semenov, I. R. Vassiliev, A. van der Est, M. D. Mamedov, B. Zybailov, G. Z. Shen, D. Stehlik, B. A. Diner, P. R. Chitnis and J. H. Golbeck, J. Biol. Chem., 2000, 275, 23429-23438.

43 O. A. Gopta, Y. L. Kalaidzidis, A. Y. Semenov and J. H. Golbeck, Biochim. Biophys. Acta, 2006, Suppl. vol. 14, 273-273.

44 N. M. Atherton, Principles of Electron Spin Resonance, Ellis Horwood, New York, 1993.

45 Y. Kandrashkin and A. van der Est, Spectrochim. Acta A, 2001, 57, 1697-1709.

46 H. Käss, P. Fromme, H. T. Witt and W. Lubitz, J. Phys. Chem. B, 2001, 105, 1225-1239.

47 M. Plato, N. Krauss, P. Fromme and W. Lubitz, Chem. Phys., 2003, 294, 483-499.

48 W. Lubitz, in Photosystem I: The Light-Driven Plastocyanin: Ferredoxin Oxidoreductase, ed. J. Golbeck, Springer, Dordrecht, The Netherlands, 2006, pp. 245-269.
49 S. A. Dzuba, P. Gast and A. J. Hoff, Chem. Phys. Lett., 1995, 236, 595602.

50 R. Bittl and S. G. Zech, Biochim. Biophys. Acta, 2001, 1507, 194-211.

51 R. Bittl and S. G. Zech, J. Phys. Chem. B, 1997, 101, 1429-1436.

52 R. Bittl, S. G. Zech, P. Fromme, H. T. Witt and W. Lubitz, Biochemistry, 1997, 36, 12001-12004.

53 S. G. Zech, W. Lubitz and R. Bittl, Ber. Bunsen Phys. Chem., 1996, 100, 2041-2044.

54 A. Savitsky, A. Dubinskii, H. Zimmermann, W. Lubitz and K. Möbius, J. Phys. Chem. B, 2011, 115, 11950-11963.

55 A. van der Est and Y. E. Kandrashkin, Appl. Magn. Reson., 2007, 31, $105-122$.

56 Y. E. Kandrashkin, W. Vollmann, D. Stehlik, K. Salikhov and A. van der Est, Mol. Phys., 2002, 100, 1431-1443.

57 C. C. Moser, J. M. Keske, K. Warncke, R. S. Farid and P. L. Dutton, Nature, 1992, 355, 796-802.

58 I. Wyndhamn, A. van der Est, D. Stehlik, R. Cohen and J. Golbeck, in Photosynthesis: Fundamental Aspects to Global Perspectives, ed. A. van der Est and D. Bruce, Alliance Communications Group, Lawrence Kansas, 2005, pp. 88-90.

59 M. Iwaki and S. Itoh, Plant Cell Physiol., 1994, 35, 983-993.

60 P. Jordan, P. Fromme, H. T. Witt, O. Klukas, W. Saenger and N. Krauß, Nature, 2001, 411, 909-917.

61 R. C. Prince, P. Lloyd-Williams, J. M. Bruce and P. L. Dutton, Method. Enzymol., 1986, 125, 109-119. 